\title{
A computerized system to monitor resilience indicators in organizations
}

\author{
Paulo Victor Rodrigues de Carvalho ${ }^{\mathrm{a}, \mathrm{b}^{*}}$, Alan Pinheiro de Souza ${ }^{\mathrm{b}}$ and Jose Orlando Gomes ${ }^{\mathrm{b}}$ \\ ${ }^{a}$ Nuclear Engineering Institute, Cidade Universitária, Rio de Janeiro, RJ, Brazil \\ ${ }^{\mathrm{b}}$ Graduate Program in Informatics-NCE\&IM, Cidade Universitária, Rio de Janeiro, RJ, Brazil
}

\begin{abstract}
The concepts developed by resilience engineering allow the understanding and monitoring the functioning of organizations and, particularly, to map the role of human activities, in success or in failure, enabling a better comprehension about how people make decisions in unexpected situations. The capture of information about human activities in the various organization levels gives managers a deeper real-time understanding of what is influencing the people performance, providing awareness of the factors that influence positively or negatively the organizational goals initially projected. The monitoring is important because the correct functioning of complex systems depends on the knowledge that people have to perform their activities and how the system environment provides tools that actually support the human performance. Therefore, organizations should look forward through precursors in operating signals to identify possible problems or solutions in the structure of tasks and activities, safety, quality, schedule, rework, and maintenance. We apply the concepts of resilience engineering to understand the organization by the analysis of cognitive tasks and activities. The aim is the development of a computerized system to monitor human activities to produce indicators to access system resilience. The validation of the approach was made in a real organization and the results show the successful applicability of the system. Based on findings obtained after the experiment of the system in a real organization, and managers and workers opinions, it was possible to show that the use of system provided an anticipated (real-time) perception about how activities are effectively being performed, allowing managers and workers to make decisions more consistent with daily problems, and also to anticipate solutions to cope with unexpected situations.
\end{abstract}

Keywords: Cognitive Tasks Analysis, performance monitoring, safety control, resilience engineering.

\section{Introduction}

Most organizations that deal with complex and dynamic situations have to cope with unforeseen and unwanted events during the execution of their activities. Such organizations are embedded in scenarios that may change without notice, influencing the organization behavior. In other words, there are transitions between different business contexts lead to variabilities that difficult the decision making process in all organization levels, from the sharp to blunt end workers.

Variabilities emphasize the potential complexity of the contexts in which organizations operate and, consequently, the people involved in the execution of tasks, from activities that require intense mental effort, when the people are often challenged to adapt dynamically their behavior and decision making to maintain organization's performance at satisfactory levels. However, when not properly monitored, such adaptations in activities may endanger the safety aspects and the organizations own business [1].

To cope with this situation, managers must have information to monitor performance variabilities, and in most organizations such information is not monitored. Instead, the information about performance variability is not perceived on time because it is scattered and fragmented in different media, including the memory of the workers themselves, which suffer interference from their own mental models and memory decay time [2],[3].

Organizations can become more resilient when managers in the various hierarchical levels have information about the activities being performed in

\footnotetext{
*Corresponding author. E-mail:paulov@ien.gov.br
} 
order to provide the resources necessary for people to adapt and make decisions in unexpected and unwanted situations, while maintaining the production flow in an effective and safe way [4].

In this article we present some concepts of resilience engineering and organizational management in other to develop a proposal to monitor resilience in organizations using a computerized support system.

\section{Resilience engineering: a new paradigm for system safety}

Traditional safety engineering usually describe accidents as a result of some failure (technical, human or organizational), something that is not expected in the normal organization performance. Although safety engineering have developed powerful models and a complete set of methods, tools and systems to analyze and improve organization safety, most of these are based on a forced linearization of complex systems. Therefore they are based in an inadequate perspective to deal with the complexity of events that occur in modern systems [5], [6].

The resilience engineering appears as an alternative to these traditional approaches to safety management. For resilience engineering failure and success are both normal results of how organizations operate in complex scenarios. Organizations must cope with variations in their functions, requiring adjustment and adaptation of people activities to respond adequately to the variability and make decisions according to the finite resources and time available in order to achieve the system goals [7].

Resilience should be seen as the ability of systems to anticipate and the ability to adapt to potential surprises and failures. Therefore, to be resilient, an organization should also monitor their ability to cope with variabilities occurring outside their standard operating model.

These findings highlight the importance to understand the distinction between how the system really works when face uncertainty (what function properly even with adaptations), and how the system was designed to recognize and deal with these uncertainty, and variability. Thus, it is necessary mechanisms for monitoring and control potential threats during normal operations, and understands how and why people make deviations beyond the perimeters considered safe in performing tasks.

The production pressure increases the appeal for successive shifts in organizational mode of operation (normalized deviations/improvisations), and situations prone for sacrifice decisions [8] characterizing the organization drift into failure process. Recognizing this situation is difficult because the entire safety and production system seems to glide along with the operating system into their safety boundaries [9], [10].

Resilience management must be based in a broad understanding of real organizational performance. Accidents reflect the unexpected combinations of normal (something that is happening, not something expected or desired) performance variability that overlap in time and mutually affect each other. The paths to this understanding, although still under discussion, have multiple approaches, many outlined in theory only, and lacking in empirical verification. Still, there are similarities in the various approaches to enhance resilience in organizations, as described below.

Westrum [11] argues that there is little doubt about the need to better understand the tasks to be performed and how and why the activities are performed in some special way. Flin [12] adds that the skills for the management of resilience must be based on the diagnosis of operating signals that point to drift to the edge of a safety perimeter.

The management of resilience seeks to establish a monitoring environment for the proactive control of safety in organizations. Thus, the factors that may interfere with the safety must be constantly accessed to prevent the occurrence of incidents/accidents. The creation of predictive indicators (that provides a proactive vision) is essential to achieve these objectives and avoid the identification of problems only after the fault detection.

\section{Indicators of resilience}

\subsection{Understanding tasks and activities}

A detailed understanding of the work in context is very important, because the variability in the various activities may combine in ways often unlikely, generating complex scenarios whose outcome is difficult to predict for managers and operators. Some approaches are based in these principles for the development of large models that can explain the dynamics of the system and how combination of these performance variations can lead to major accidents [13],[14].

Hollnagel [13] proposes a model to understand the socio-technical systems based on system functions. 
The approach relies on complexity theory and argues that systems cannot be decomposed into parts, because they are non-linear. System operation can only be adequately described considering relations and resonances among its functions. In the Leveson's [14] model the systems and their components are kept in a state of dynamic equilibrium by repetitions of information feedback and control loops. The approach relies on the dynamic systems theory. In her model accidents or losses results from the lack of safety control in the design and operation of complex sociotechnical systems. Both approaches are quite powerful, however, they are more focused on the investigation of accidents and risk management. They require a broad theoretical knowledge of people and a considerable time to organizations in their deployments.

In contrast to previous approaches, other studies rely on practices less complex and faster application. These practices use Cognitive Task Analysis (CTA) to study a variety of work domains from different perspectives [15]. CTA is a set of methods used to investigate cognitive processes in workplaces conducted by different human operators in handling and technological artifacts. The methods seek to understand how human agents work and see how they give meaning to events. The aim is to capture the strategies of decision making, in which workers pay attention, to what they may be thinking at the time of action, and what they know about the process.

\subsection{Indicators development}

Various studies have said that the development of indicators for assessing the resilience should start by knowledge of organizational processes [16], [17]. However, there are few systematic attempts to create it. The difficulties lie not only in design, but in limited opportunities for validation on real events [18].

The organizational safety indicators traditionally used are obtained after the occurrence of unwanted events (lagging indicators). However such indicators e.g. frequency of accidents, do not provide sufficient knowledge to prevent future events, or may become obsolete when the time for analysis is too long. Thus, there is a need to develop predictive or leading indicators to access resilience. The two types of indicators must coexist, since both contribute in providing relevant information to organizations, but they have different origins and objectives: the first focus the results of activities, while the second on how and why activities are performed.
The design of useful indicators is not a simple task because it involves several aspects such as capture, organize, evaluate, visualize and analyze data, and make decisions about the event to be measured, in addition we also need adequate metrics, simple evaluation, calculation and analysis [20].

After defining the indicators, the next step is the establishment of metrics. This is very important, because these metrics should depict the correct indicators properties. Otherwise, the result of analysis will become costly and often delivering unrealistic conclusions. Despite extensive studies on the definition of indicators measurement scales [21], the paths to this development in the field of resilience are rarely discussed. Huber, [22] and EPRI [23] use ordinal scales as a metric to measure resilience.

The capture of information for indicators development used is normally based on questionnaires, as they allow access to a wide audience and after the evaluation of indicators, the results are presented through graphs that summarize information captured during the evaluation [22],[23]. Some evaluation methods use heuristics (estimated weights and weighted average) before present final results [24].

Recent literature talks a lot about dashboards. The term is used to indicate a panel indicator providing immediate information that illustrates the performance of the entire organization. The data are generated for the top level management that requires a broad view of business [25].

Regardless of the means used in the analysis of the resilience, the organization management should have quick and clear information about the main aspects of the evaluation, i.e., perception of relevant information collected during the evaluation, in order to display trends on the dimensions investigated, alone or compared with others.

\subsection{Information systems}

One goal of resilience engineering is to develop artifacts to provide information to support decision making processes to ensure that organizations remain within safety margins. Although the characteristics of these systems need to be specified, highlights the need for understanding the work as it is really performed, and the organizational perception on how the performance impact safety and the resources that are available for actions to deal with disturbances (buffer capacity).

The main information system developed to indicate resilience found in the literature with similar 
goals to ours is the Proactive Assessment of Organizational and Workplace Factors (PAOWF) [23]. The system provides information that allows management to access how workplace factors influence operational activities, without the occurrence of unexpected or unwanted event. PAOWF was designed to be used in nuclear power plants, and it is based on the idea that the most effective way to find out people obstacles and/or need to adapt the work to cope with daily is to ask them. Evaluations occur by questionnaires on organizational indicators established for the work domain. The PAOWF does not specify evaluation factors, since these should be customized for plants and working groups being analyzed. The evaluations are anonymous to encourage participation and enable the capture of information about how the work is really done.

\section{The proposed approach to monitor resilience indicators}

The approach is based on the detection of variabilities in normal (daily) working conditions in the operational levels, because in this layer of an organization lies information relevant to the development of leading performance indicators. Another feature of the system is to facilitate its use in order to get more data. Using the system an organization can get information to control variabilities production processes without requiring extensive theoretical knowledge and time of final users.

\subsection{Cognitive work analysis}

Cognitive Work Analysis (CTA) methods and tools are needed to understand how workers actually behave in an organization. This goal can be achieved by conducting field studies that record the practical challenges that people face at work, and practices that workers develop to cope with complexity [2],[3]. CTA allow a comprehensive understanding of the work situation, as all the aspects of the work can be studied and considered at the time of its completion.

Data collection occurs through interviews, direct observations, walk and talk through, as these techniques provide ways to capture cognitive information, especially when they are combined, maximize advantages and minimize limitations of single technique use.

The CTA in collective work settings should considers different aspects: human agents, highlighting their profile, how they carry out tasks and interact to achieve their goals, the work environment and technical systems, showing functions and their dependencies. Figure 1 highlights some of the dimensions of analysis necessary to understand the different types of dimensions that shape the behavior of workers.

Thus, the analysis should consider factors that generate impacts and deviations in the performance. The understanding of real work in collective work settings (group success or failure to reach their goals in real situations) involves the analysis of groups of individuals, rather than the study individual cognition.

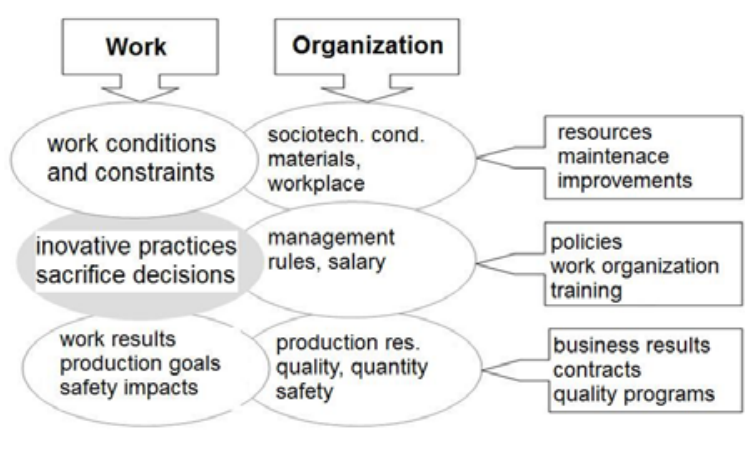

Figure 1 Work analysis dimensions.

\subsection{Resilience indicators}

The indicators should reflect a set of organizational factors that influence the performance of organizational activities. Resilience indicators were defined based on resilience engineering research and on safety culture indicators used in nuclear domain [23], [26]. These works have an initial set of 10 factors: communication, resources and equipment, group activities and interfaces, condition of materials, planning and scheduling, policies, procedures and documentation, roles and responsibilities, structure of tasks, training and experience.

In order to be applied in a specific organization there is a need to refine the indicators in new components (criteria and aspects). The breakdown should reflect properties of the activities and the characteristics of resilience in the activities performed by the worker. Figure 2 highlights the refinement of an indicator in criteria and aspects. 


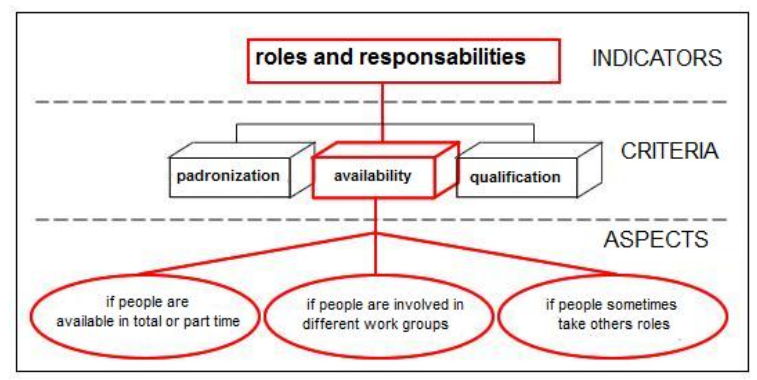

Figure 2 Indicators' refinement process.

This refinement when properly done increases the knowledge about the conditions that influence the resilience of activities being performed. The working conditions considered involve elements of organizational, human, technological, physical, political and economic dimensions. The elements are related to each other in a non-linear way and may change according to the socially constructed situation of an organization.

After define the structure and perform the validation of elements, the indicators became available to operators so that they can evaluate them according to the criteria and aspects that were found to be more relevant to carry out their activities. The final evaluation of each indicator will compose a panel that represents the resilience of the organization.

The final evaluation of the indicators, the elements that are present in the higher abstraction level in the components structure is performed in an indirect way, using the elements present in lower abstraction levels, i.e., the criteria and aspects. The assessment takes place by a questionnaire that people should respond during their daily activities. The aim is the generation of continuous data that reflect the feeling (satisfaction or disappointment) of people doing their work. The data collected are available online for the management, promoting awareness about the issues that affect the performance and their safety impacts. Figure 3 shows part of the resilience indicators panel of an organization after 3 days of analysis, in which 3 different work teams perform the same activities.

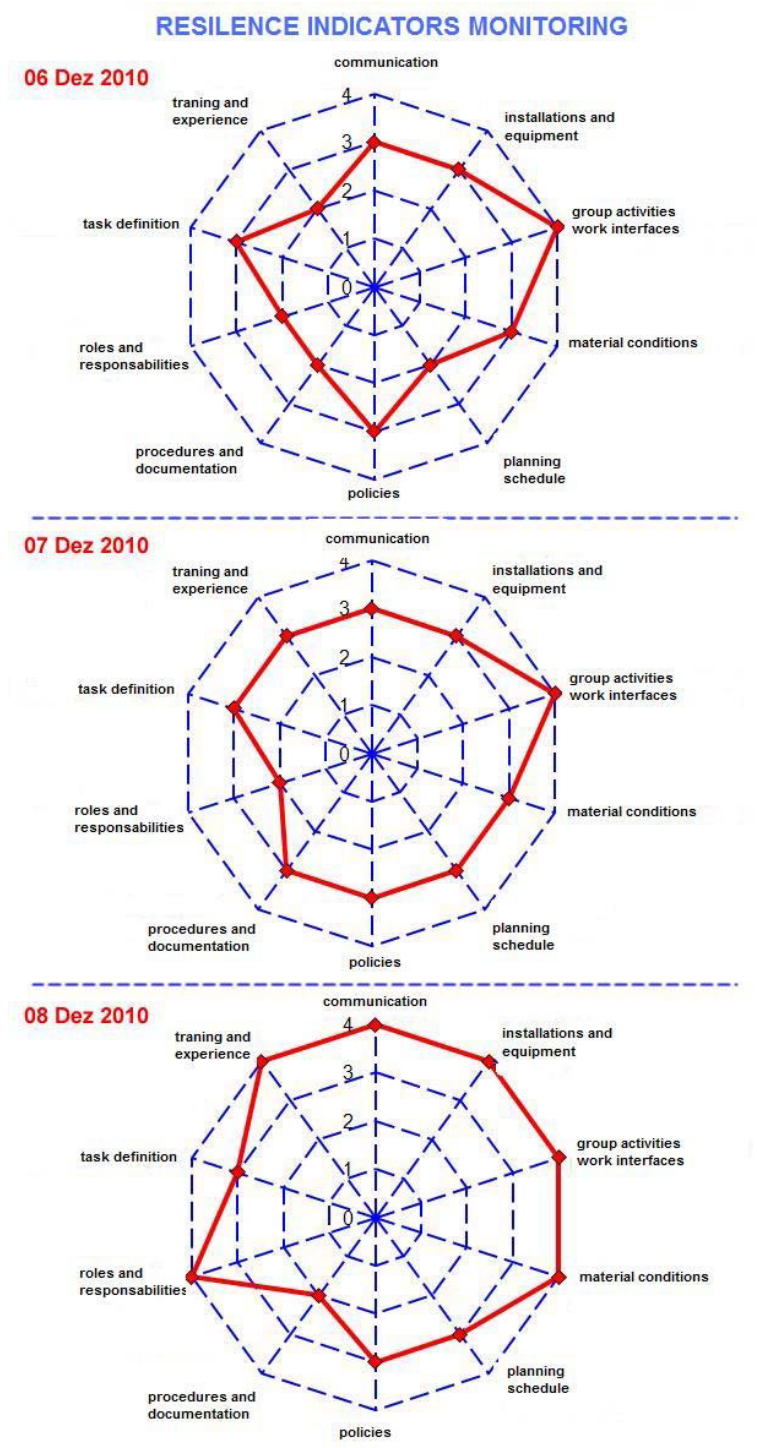

Figure 3 Radial graphs as a panel of resilience indicators.

The monitoring of indicators allows managers to have a big picture about how the activities were/are being held throughout the work day and a dynamic perception of the what kind of problems people have to perform their tasks. As shown in figure 3, the monitoring results can be displayed by radial graphs that provide management a multidimensional view of the model, or by trend graphics that allow an analysis of the evolution of an indicator over time. The organizational assessment can be applied whenever management wants information about the factors that influence the task performance or to check the status of tasks after changes in the work situation. However, we believe that the assessment should be continuous 
so that managers are always monitoring the active and latent threats, since most organizations are not aware of these threats.

\section{An information system to monitor resilience in organizations}

The information system collects data on organizational aspects that influence the operational activities and allows management awareness of these issues. The system includes aspects and indicators that are defined according the work situation under analysis, using CTA methods and techniques. The system allows anonymous records and comments on the aspects that influence the work, and are keyed to guide the evaluation and further (deeper) analysis of work constraints and variabilities.

The information system consists of three modules. The first is the configuration module (restricted to administrators) in which the basic information are defined. The second module is used by people to evaluate indicators in the various levels of the organization. The final module allows the analysis of data (free access to managers). The basic architecture is sketched in Figure 4.

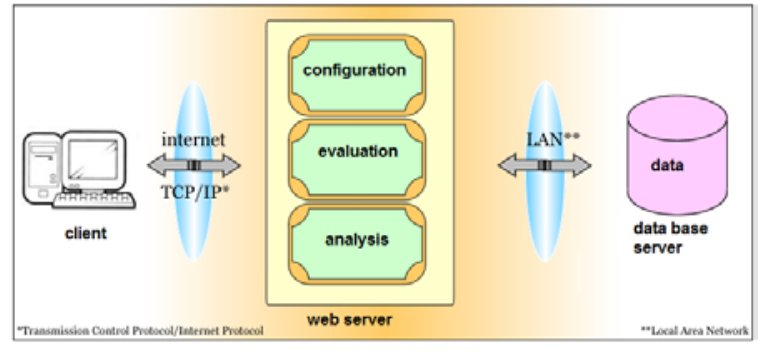

Figure 4 Information system.

The computer system was named Access Organizational Resilience (AOR). It is designed to be used and implemented in the web programming language PHP and uses the MySQL database to store data.

\section{Conclusions}

The paper proposes an approach for assessing resilience in organizations by identifying the main factors involved in people tasks and activities and how these are affected during daily work. The awareness of such information helps management to make decisions supported by larger and more realistic know- ledge of how and why activities are being performed according to the view of sharp end operators. The approach is based in principles pointed out in much resilience engineering studies that points out to the need of resilience indicators. We develop an information system to support the monitoring of resilience indicators in organizations that provides a technological opportunity to detect working constraints and deviations towards organization safety boundaries, creating predictions about changing patterns of risk before failures actually happen.

\section{Acknowledgements}

The authors gratefully acknowledge the support of the Brazilian Research Council (CNPq) and of Rio de Janeiro Research Support Foundation (FAPERJ).

\section{References}

[1] L. Carver, M. Turoff, Human-Computer Interaction: The Human and the Computer Emergency Team in the Management Information Systems. Communications of the ACM, New York 50, 3, (2007), 33-38.

[2] P. V. R. Carvalho, I. L. Santos, J. O. Gomes, M. R. S. Borges, Micro incident analysis framework to assess safety and resilience in the operation of safe critical systems: a case study in a nuclear power plant. Journal of Loss Prevention in the Process Industries 21, (2008), 277-286.

[3] P. V. R. Carvalho, M. C. Vidal, E. F. Carvalho, Nuclear power plant communications in normative and actual practice: A field study of control room operators' communications. Human Factors and Ergonomics in Manufacturing 17, (2007), 43-78.

[4] E. Hollnagel, Safety Management - Looking Back or Looking Forward, in: Remaining Sensitive to the Possibility of FailureResilience Engineering Perspectives, E. Hollnagel, C. Nemeth, S. Dekker, Ashgate Studies in Resilience Engineering, 2008, pp. 63-79.

[5] T. Sheridan, Risk, Human Error and System Resilience: Fundamental Ideas, Human Factors, 50,3, (2008), 418-426.

[6] J. Wreathall, Properties of Resilient Organizations: An Initial View, in: Resilience Engineering: Concepts and Precepts, E. Hollnagel, D. Woods, N. Leveson, Aldershot, UK: Ashgate, 2006, pp 275-286.

[7] E. Hollnagel, D. Woods, N. Leveson, Resilience Engineering: Concepts and Precepts, Aldershot, UK: Ashgate, 2006.

[8] J. O. Gomes, D.D. Woods, P.V.R., Carvalho, G. Huber, and M.R.S. Borges, Resilience and brittleness in the offshore helicopter transportation system: the identification of constraints and sacrifice decisions in pilots' work. Reliability Engineering \& Systems Safety, 94, (2009), 311-319.

[9] S. Dekker, Resilience Engineering: Chronicling the Emergence of Confused Consensus, in: Resilience Engineering: Concepts and Precepts, E. Hollnagel, D. Woods, N. Leveson, Aldershot, UK: Ashgate, 2006, pp. 77-90.

[10]J.Rasmussen, I. Svedung, Proactive Risk Management in a Dynamic Society. Karlstad, Sweden: Swedish Rescue Services Agency, 2000. 
[11]R. Westrum, A Typology of Resilience Situations, in: Resilience Engineering: Concepts and Precepts, E. Hollnagel, D Woods, N. Leveson, Aldershot, UK: Ashgate, 2006, pp. 55-66.

[12]R. Flin, Erosion of Managerial Resilience: Vasa to NASA, in Resilience Engineering: Concepts and Precepts, E. Hollnagel, D. Woods, N. Leveson, Aldershot, UK: Ashgate, 2006, pp 223-233.

[13]E. Hollnagel, Barrier Accident Analysis and Prevention. Aldershot, UK: Ashgate, 2004.

[14]N. Leveson, A New Accident Model for Engineering Safer Systems, Safety Science, 42, 4, (2004), 237-270.

[15]B. Crandall, G. Klein, R. Hoffman, Working Minds: A Practitioner's Guide to Cognitive Task Analysis. The MIT Press, Cambridge, Massachusetts, 2006.

[16] J. Wreathall, Measuring Resilience, in: Preparation and Restoration-Resilience Engineering Perspectives, E. Hollnagel, C. Nemeth, S. Dekker, Ashgate Studies in Resilience Engineering, 2009, pp. 95-114.

[17]EPRI, Final Report on Leading Indicators of Human Performance, Electric Power Research Institute, 2001.

[18]D. Mendonça, Resilient Measures of Performance. in: Remaining Sensitive to the Possibility of Failure-Resilience Engineering Perspectives, E. Hollnagel, C. Nemeth, S. Dekker, Ashgate Studies in Resilience Engineering, 2008, pp. 29-47.

[19] M. Grabowski, Leading Indicators of Safety in Virtual Organizations, Safety Science, 45, (2007), 1013-1043.
[20]EPRI, Business Performance Indicators for Nuclear Asset Management, Electric Power Research Institute, 2006.

[21] M. Boudreau, D. Gefen, D. Straub, Validation in Information Systems Research:The State of the Art Assessment. MIS Quarterly, 25, 1, (2001)1-16.

[22]G. Huber, A Method for Assessing Resilience in Complex Organizations: A Study in Helicopter Aviation, MSc Dissertation, Graduate Program in Computer Science, Federal University of Rio de Janeiro, 2010.

[23]EPRI, PAOWF Users' Guide, Electric Power Research Institute, 2000.

[24]R. Senra, A Reference Model for Capacity Evaluation of Emergency Response Organizations, MSc Dissertation, Graduate Program in Computer Science, Federal University of Rio de Janeiro, 2007.

[25]N. Rasmussen, C. Chen, M. Bansal, Business Dashboards: A Visual Catalog for Design and Deployment, John Wiley \& Sons, 2009

[26]INPO, Human Performance Fundamentals Course, Institute for Nuclear Power Operations, 1997. 